\title{
Implementing tobacco interventions in the real world of managed care
}

Jack F Hollis, Richard Bills, Evelyn Whitlock, Victor J Stevens, John Mullooly, Ed Lichtenstein

Over the years we have been working to develop, test, and implement tobacco control interventions as a part of routine care within Kaiser Permanente. Most of our work has been in Kaiser Permanente's northwest division, based in Portland, Oregon, but we have also implemented similar approaches in several other divisions, including Ohio, Hawaii, and Georgia. I will first describe our general approach, which we call the TRAC model ("tobacco reduction, assessment, and care"), and then share both our progress and some very real difficulties we have encountered in trying to implement the program throughout the health care system.

The rationale for delivering brief tobacco intervention during routine care is familiar to those who work in cessation. ${ }^{1}$ Tobacco remains the most important cause of preventable disease. We know that most smokers see clinicians frequently, and that these visits create teachable moments when patients are receptive to advice and intervention. When we routinely ignore these intervention opportunities, we are, in effect, failing our patients. Indeed, metaanalyses from the Agency for Health Care Policy and Research (AHCPR) clinical guideline ${ }^{2}$ show that brief advice and support lead to modest but consistent long term effects on smoking cessation. We also know that brief tobacco interventions are among the most cost effective of all medical care procedures we routinely offer. ${ }^{3}{ }^{4}$ It is for these reasons that the Health Plan Employer Data Information Set (HEDIS) and other quality monitoring groups are holding health care systems accountable for addressing tobacco during clinical care. For me, however, the most important reasons to offer cessation advice and assistance are that our patients want, need, and expect this kind of support.

How are we doing as a nation in delivering cessation advice during medical care visits? Figure 1 displays time trend data ${ }^{5}$ from the National Household Interview Survey ${ }^{6-8}$ and the Current Population Survey from the US Bureau of Labor Statistics. ${ }^{5}$ Both surveys use national probability samples to estimate the percent of smokers with visits in the preceding year who report that a physician has ever advised them to quit smoking. Back in 1974, few smokers reported ever receiving cessation advice. Advice rates rose sharply by 1986 and have continued to slowly improve up through the mid 1990s.
Elsewhere, I have projected what impact our current clinician advice rate might have on smoking cessation rates ${ }^{5}$ among the 35 million smokers who see a clinician each year. I assumed a 3\% spontaneous quit rate and that primary care interventions, when they are delivered at all, largely consist of simple advice to quit. ${ }^{9}$ We might call this the $2 \mathrm{~A}$ model ("ask and advise"), as opposed to the $4 \mathrm{~A}$ model ("ask, advise, assist, and arrange") recommended by the National Cancer Institute (NCI) and the AHCPR. Meta-analyses from the AHCPR clinical guideline ${ }^{2}$ estimate that the cessation odds ratio for one to three minutes of simple brief advice is 1.2. Delivering brief advice to $60 \%$ of the smokers who see a clinician each year across the country might generate about 126000 additional quitters over and above the spontaneous rate. If we increase the simple advice rate to $90 \%$, we would produce something like 189000 additional clinician generated quitters, which would be a substantial achievement. But suppose, once each year, clinicians advised $90 \%$ of smokers and that, for the half of these smokers who are at least considering quitting at any given time ("contemplators" in Prochaska's model), clinicians or their staff also provided 10 minutes of actual cessation counselling and assistance. The AHCPR metaanalyses estimate that 10 minutes of cessation assistance yields a much higher 2.4 odds ratio. This would yield about 756000 additional quitters per year or a sixfold increase in the number of clinician generated quitters over current practice. My point here is that the third and fourth $\mathrm{As}$ in the $4 \mathrm{~A}$ model really do matter, and we need to overcome the very real barriers that are preventing the delivery of

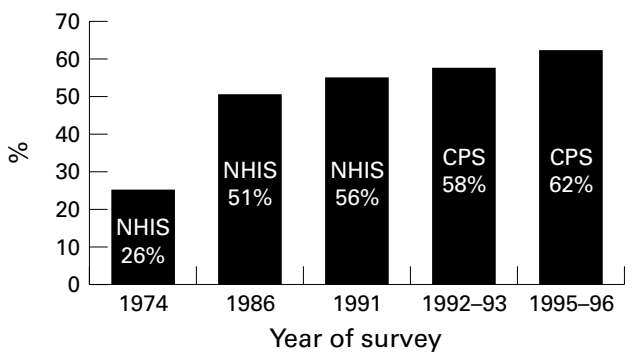

Figure 1 Percentage of smokers reporting ever receiving physician advice. NHIS, National Household Interview Survey; CPS, Current Population Survey. 
systematic and comprehensive tobacco intervention in most primary care settings today.

The most fundamental barrier is that tobacco intervention is simply not part of the clinical culture, ${ }^{10}$ and this gets expressed in a variety of ways. I have often heard clinicians express a reluctance to "badger" patients about lifestyle choice, and many appear to feel on shaky ground when addressing the issue unless the patient presents with a serious smoking related condition. Many clinicians also simply lack the cessation skills, confidence, and comfort level to raise the issue with smokers, who are almost inevitably ambivalent about quitting. Another problem is that many clinicians and health care system managers are not convinced that brief interventions really increase quit rates, or that patients will still be a part of the health care system by the time any benefits of cessation occur. Health care systems grow out of and reflect this same clinical culture in which tobacco intervention is not viewed as a priority. As a result, most health care systems allocate little or no time, funding, support staff, or system planning to ensure that the job gets done. For example, rarely do we see tobacco control performance goals, tobacco use registries, organised reminder systems for staff, performance monitoring, or performance feedback for tobacco control activities. Even more rare are examples of reimbursement or incentive programs to improve practice patterns in this area.

\section{The TRAC model}

Some years ago my colleagues and I began asking ourselves what it would take practically in order to implement the four As in a complicated, busy, managed care environment like Kaiser Permanente. We knew that the 4A model was clearly efficacious. We also knew that few physicians were delivering the four As systematically, and that asking busy clinicians to regularly spend 3-5 minutes of the 10 minutes they have with the patient was just not going to work as a general rule. Our solution was to develop TRAC, which is merely a team approach for delivering the standard $4 \mathrm{~A}$ model (fig 2). The roles for various staff are as follow.

TOBACCO ASSESSMENT

Clinical assistants identify and document the tobacco status of every patient during intake, and alert the clinician when they have a smoker or a recent quitter. Recent quitters are those who have quit within the last six months who might need reinforcement, encouragement, or relapse-prevention support.

\section{CLINICIAN ADVICE}

Clinicians are encouraged to do all they can within the time available, but at a minimum to give a clear, yet respectful, 30 second advice message that might go something like this: "Stopping tobacco use is the single most important thing you can do to protect your health, particularly because of your [condition]. As your clinician, I strongly advise you to stop soon, but I know that has to be your choice. I believe you can do it, but only when

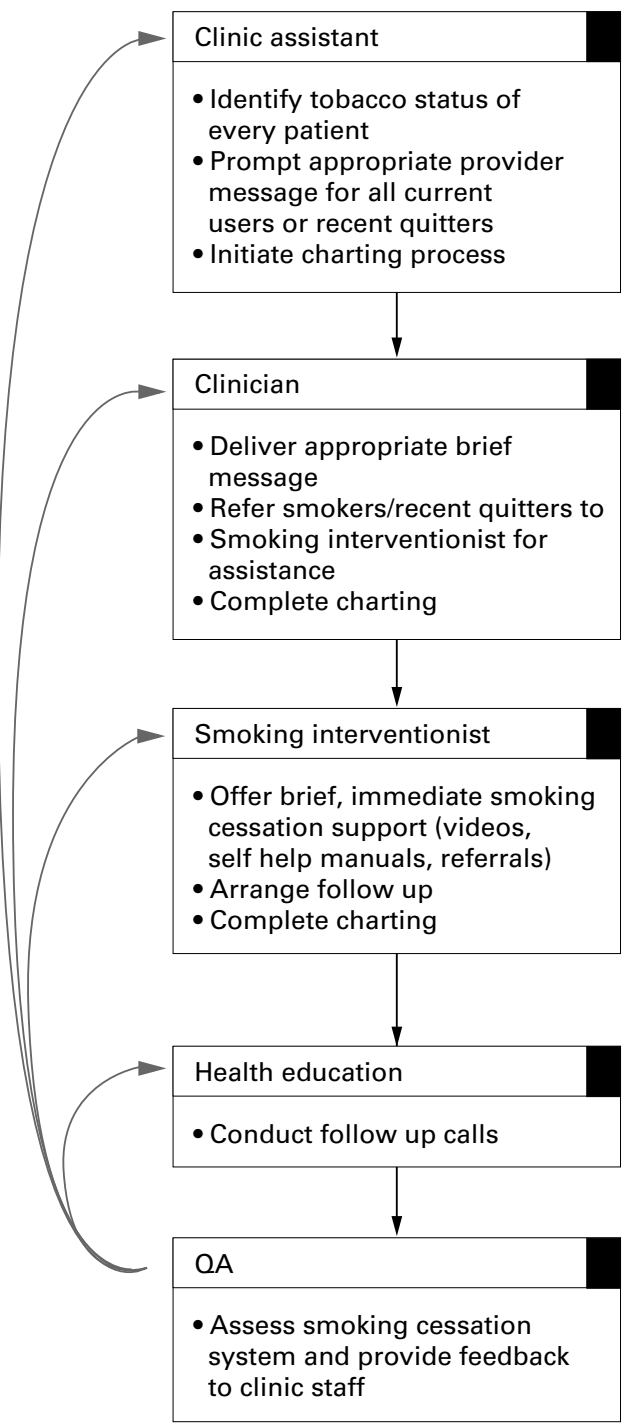

Figure 2 TRAC (tobacco reduction, assessment, and care) clinic flow outline, office based smoking cessation system.

you are ready to try. So tell me, are you seriously thinking about quitting in the next six months?"

This kind of message gives clear advice tailored to the patient's specific symptoms and risk factors. It also acknowledges the patient's choice and responsibility in making this important lifestyle choice. The clinician also expresses confidence that the patient can succeed, once they put his/her mind to it. The last piece of the advice message is a triage question. If the patient is not seriously thinking of quitting, the clinician offers a pamphlet discussing the pros and cons of cessation, and arranges to check back with the patient at the next visit. If the patient is at least considering quitting in the next six months, the clinician refers them to a nurse or someone else in the clinic who can provide additional assistance at the end of the visit.

NURSE ASSISTANCE

The goal of the nurse delivered assistance component is to help the patient build their motivation and confidence, and take the next steps toward cessation. We start by having the 
patient watch a nine minute video we developed that deals with common concerns, barriers, and questions. The nurse then returns and determines if the patient is ready to take action (for example, set a quit date), if they are interested in group intervention or pharmacological treatment, or if they have other concerns, questions, or needs.

\section{FOLLOW UP SUPPORT}

If the patient picks a specific quit date, the nurse arranges to have a health educator call at a convenient time a day or two after the quit date to provide support and encouragement. Ideally, the same nurse would make the call, but we felt it was more practical to have this done centrally by experienced smoking counsellors in our health education department. Although we reserve these follow up calls for patients who actually set quit dates, we feel they are very useful. First, they provide a social incentive for the patient to actually make a quit attempt on the appointed day. Secondly, patients seem to really appreciate the caring and support during the initial and most difficult part of the quitting process. Finally, if the patient is having difficulty, the counsellor can use the opportunity to encourage a new quit date and/or enrollment in a more intensive support program.

QUALITY MONITORING

The final component of TRAC is a quality assurance process, which, in my mind, is essential to changing and maintaining staff practices. The goal should be to monitor each component of the program that you believe is instrumental to its success (for example, asking, advising, assistance, follow up calls, etc).

\section{STAFFING DEMANDS}

When I describe this model, people often say: "Gee, this is great, but it sounds expensive. We don't have the staff or resources to do this in our setting." My response is that this does require some expense-it is certainly not free-but it is also not nearly as expensive as many might imagine. Let us assume that a full time primary care clinician has about 30 appointments a day, that $27(90 \%)$ of those patients show up, and that 24 (or 90\%) are assessed for smoking status. In our population, about five $(20 \%)$ of these patients will use tobacco. If clinicians gave advice messages to four of them $(80 \%)$, they would be doing really well. Four, 30 second messages per day add up to two minutes per day for a full time clinician. This is really not much, considering that tobacco is the number one public health problem of our time. Most clinicians are probably already spending at least this much time on more lengthy discussions with occasional smokers, so this model may actually save them some time while allowing them to intervene with more smokers in a typical day.

How about the nursing time? Maybe two $(50 \%)$ of those four advised smokers will be seriously considering quitting in the next six months. Even if they were not necessarily ready to set a quit date today, we would encourage them to see the nurse. Not all would, of course, so maybe only one or two of these motivated patients would spend about three extra minutes with a nurse each day. Of patients who see the nurse, about one in three will set a quit date and need a follow up call. The calls usually average about five minutes. In summary, a very liberal estimate of what this takes is about two minutes of clinician time and six minutes of nurse (or other staff) time for each full time clinician. Calling time, a video player, training, and a performance monitoring/reporting mechanism are other costs to be considered. So there are costs, but they are really not very large compared to other quality improvement efforts we are doing for other medical procedures that are far less cost effective.

\section{TRAC efficacy trial}

We tested the TRAC model in a randomised trial $^{1112}$ and found that the nurse assisted components nearly doubled the long term quit rate compared to a 30 second clinician advice message alone. The program was equally effective for men and women. ${ }^{13}$ Most importantly, the team approach made it practical for busy clinicians to raise the tobacco issue routinely with most of the smokers they see in a day. Our initial study was really a test of the efficacy of the approach when delivered under favourable and well monitored research conditions. The next and much more difficult step was to conduct a system level implementation trial to test the effectiveness under real world conditions.

\section{TRAC dissemination trial}

We are now conducting a dissemination trial to see if TRAC can be implemented on a much larger scale in randomly selected primary care offices and whether this team approach will work in the real world on an ongoing basis. We began building our health plan's commitment to the effort back in 1993 when we first decided to seek funding from the National Institutes of Health to evaluate the dissemination effort. We sought and obtained letters of support from our medical director, the chiefs of medicine, the physicians in charge of the 10 primary care facilities, the director of quality medicine, and a variety of other people. Support was relatively easy to obtain because staff and clinicians reported positive experiences with the TRAC model during our original study.

TOBACCO TASK FORCE

Once our grant was funded, we convened a tobacco clinical quality improvement task force, which was made up of 16 people, including physicians, managers, nurses, pharmacists, health educators, and one researcher $(\mathrm{JH})$. We met about 12 times over a year and used a clinical quality improvement approach to think about how to integrate smoking intervention into various clinical settings (for example, primary care, hospitals, prenatal, etc) and from a broad variety of perspectives. The group concluded that the TRAC model was particularly 
appropriate for primary care. The group refined and tailored the general approach and developed an implementation plan to guide the roll out process. The plan was then considered and approved by a series of relevant health plan committees, including the prevention steering committee, the clinical quality planning counsel, and finally, the regional operations group that oversees all clinical functions in the system.

FACILITY LEVEL BUY IN

We now had high level approval from the organisation and some degree of legitimacy, but we still needed to sell the plan to the relatively autonomous leadership group for each of 12 primary care facilities. We made presentations to each group of facility managers to explain the program and gain their support. We also asked for their consent to assign them randomly to implement the program either right away or on a delayed basis within a randomised facilities design. Two facilities chose not to participate. One declined because they did not want to wait if they were assigned to the control group. The other one declined because they felt they could not implement the program right away if so randomised. Once a facility was randomised to "early implementation", we began working with the management team in earnest to identify and overcome the perceived barriers to implementation, and to tailor the program to the organisational, staffing, and physical characteristics of each facility. Even in a unified staff model health maintenance organisation (HMO) such as Kaiser Permanente, it is very important to attend to these site specific differences.

STAFF TRAINING

The next step was to train the staff in each facility. The training sessions were brief because that is all the time that we could get. Typically, we showed a 12 minute training video that we developed which describes the overall approach, models various staff roles, and shows how to deal with some typical patient responses. We trained the clinical assistants in one hour meetings to assess smoking status, prompt clinicians, and document. We trained the clinicians in their brief and relatively structured role during 90 minute meetings over lunch. The nurses and licensed practical nurses who would be doing the assistance component needed to have a good deal more flexibility and comfort in dealing with a broad range of smoking cessation issues. Although their role was still relatively brief and structured, we also wanted to give them some of the basic tools associated with the motivational interviewing ${ }^{14}$ and brief negotiation ${ }^{15}$ approaches. We conducted 3-4 hour workshops for the nurses and did a lot of role playing about how to work with smokers with different kinds of issues and at different levels of readiness. All the smokers referred to nurses had at least expressed some interest in quitting.
START UP AND MAINTENANCE ACTIVITIES We generally implemented the program within one day or at most two or three days following the training of staff. We wanted to start while the training was fresh and enthusiasm was high. Many nurses (and some clinicians) were nervous about addressing tobacco, and it was important to have the implementation team present in the facility initially. Two or more team members were available to provide encouragement, solve problems, debrief intervention sessions, and occasionally cocounsel a smoker with a staff member. The clinicians, clinical assistants, and nurses were all busy seeing patients while this was happening, so even small problems (for example, locating a self help manual) could easily become large barriers during this initial period. I think our primary role, however, was to be cheerleaders and to hold their hands a bit. Counselling smokers can be a little frightening if you have never done it. As staff got comfortable with the process, less and less support was needed, but someone from the implementation team was generally available for up to six weeks or so. We also provided feedback regularly at module meetings, put progress reports in the facility staff newsletters, and helped with the ongoing monitoring by the regional tobacco oversight committee. Later, we did some retraining in selected facilities, especially those that went through major reorganisations and staffing shifts.

PROGRAM EVALUATION

Changing practice patterns in busy medical settings is very difficult. We randomised eight primary care departments, which actually included 10 different facilities of 20-40 clinicians each. Before randomisation, we paired up the eight departments by matching them on location, size, and baseline tobacco advice rates as determined from a preimplementation patient survey. We then randomly assigned departments within each pair to implement the program "earlier" or "later" (our wait list control group). Our primary measures of the impact of implementation on tobacco service delivery were derived from surveys of over 20000 randomly selected patients who were seen in the "early" and "later" departments. We sent the surveys out within a few days of the patient's visit, and, with multiple mailings and a phone follow up, we achieved a $76 \%$ response rate. We asked respondents who smoked $(\mathrm{n}=2554)$ what services they received and some visit satisfaction items. We also attempted to get a more direct measure by asking staff to use a "tobacco intervention checklist" to document the services they provided to every tobacco user seen. Initially, this consisted of a three part insert that could be pasted into the chart notes (fig 3). Staff simply checked off whether they gave advice, whether the nurse saw the patient, and whether the patient set a quit date and needed a follow up call. One copy was routed to the health education department to prompt telephone support calls, one was used for data entry and monitoring, and one copy stayed in 


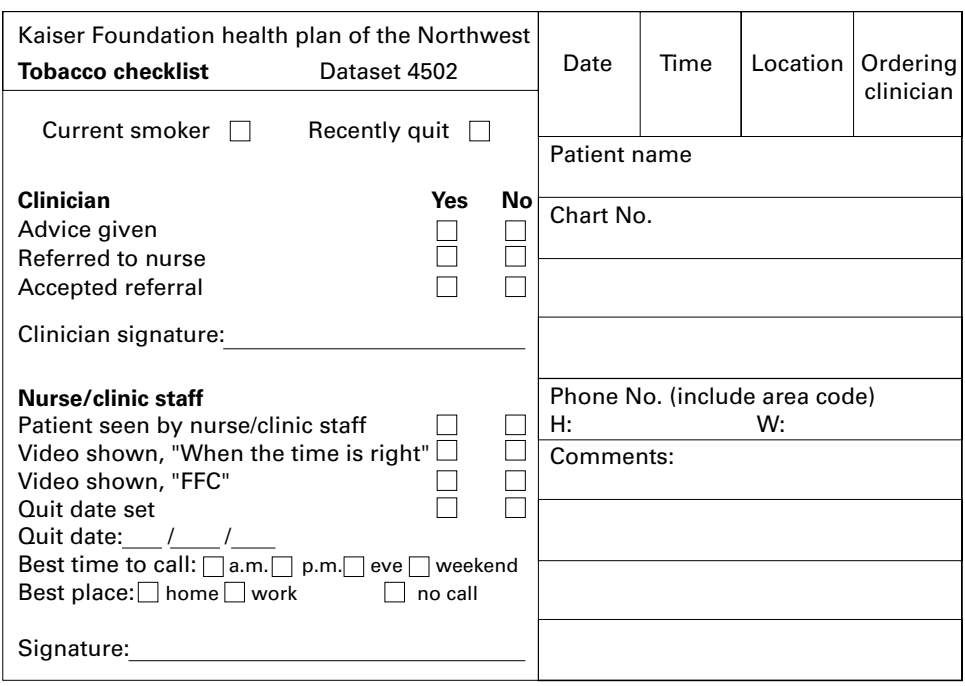

Figure 3 Tobacco intervention checklist used to document services provided to every tobacco user seen in the TRAC dissemination trial.

the medical record. In the real world, however, things happen. In the midst of the implementation process, our health plan rolled out an electronic medical record that made the paper charts obsolete. We therefore integrated the tracking and documentation process into the electronic medical record, but staff were in a learning mode for sometime and the validity of the electronic process data is questionable. We did collect reasonably good data from the paper based tobacco intervention checklists for the first three intervention facilities. What we observed was that staff used the tobacco intervention checklists fairly consistently for the first few months. Staff had little incentive to monitor what they were doing, however, and once the implementation team left the facility, documentation of tobacco services extinguished rapidly. As a result, the post-visit patient surveys provide our most reliable data on the degree to which practice patterns changed in treatment relative to control facilities.

POST-VISIT SURVEY RESULTS

The survey showed that, among primary care patients with a recent visit, the prevalence of smoking was about $18 \%$. Interestingly, those patients $(75 \%)$ who responded to the mailings had a smoking prevalence of $15 \%$. The more reluctant respondents whom we had to interview by phone were far more likely to be smokers $(26 \%)$. This has implications for HEDIS and other groups interested in using smoking prevalence as an indicator of quality. Among other problems with this measure, it appears that prevalence may be directly related to response rate. Plans with a low response rate might appear to have a lower tobacco use prevalence. High response rates are needed to get valid measures of smoking prevalence.

Preliminary results from our survey show that TRAC implementation had a significant, but relatively modest, effect on the proportion of all patients, both smokers and non-smokers, who recalled being asked about tobacco use $(60 \%$ v 54\%, respectively) at their last visit.
Among the smokers, the assessment rate was higher and the treatment versus control difference was considerably larger ( $86 \%$ v $76 \%$ ). Perhaps the clinical assistants simply do not bother to check in with patients they know are non-smokers, or maybe smokers are just more likely to recall being asked. I suspect that both might be true.

We were surprised and really pleased that $59 \%$ of smokers in the control group reported that a doctor or nurse talked to them about stopping smoking at their recent visit. That is quite impressive for the control group. TRAC implementation, however, substantially increased smokers' recall of clinician advice at a specific visit to $72 \%$.

But were these patients getting anything other than just advice? Among smokers who reported receiving advice, we found that only $18 \%$ of controls reported that they also received some indicator of even minimal cessation assistance. Usually this assistance consisted of a group cessation brochure, but some also reported receiving a self help manual, setting a quit date during the visit, or discussing pharmacological treatment. Basically, controls were getting little more than just advice. Smokers who had been advised to quit in the TRAC facilities were twice as likely $(36 \%)$ to recall receiving some form of assistance. As perhaps $50 \%$ of advised smokers would be "contemplators" who would be referred to the nurses for such assistance, this finding is fairly respectable. Many reported receiving brochures for the group cessation program (28\%), but $20 \%$ also reported watching a cessation video, $13 \%$ received a cessation manual, and $7 \%$ reported setting a specific quit date during the visit.

We also asked patients if they thought that clinicians and nurses should routinely encourage smoking patients to quit and offer assistance to those who want to quit. We were concerned that maybe smokers would resent this type of intervention, but we found exactly the opposite. Almost all smokers in both the treatment and control conditions $(97 \%$ and 95\%, respectively) agreed that clinicians and nurses should encourage and help with cessation efforts. Most $(70 \%)$ felt that staff should do this "always" or "usually."

We also looked at satisfaction with the overall visit and the clinician to see if TRAC might have had either an adverse or beneficial effect. We created a scale using three, five point visit satisfaction items $(\alpha=0.94)$. We found that smokers who recalled receiving advice were significantly more satisfied with the visit than smokers who did not recall receiving advice. The same was true for smokers who received some form of cessation assistance compared to those who did not.

In summary, the TRAC model was initially well received by staff. Patients responded when the intervention was offered, and intervention was associated with higher visit satisfaction. We also saw that intervention fell off dramatically at repeat visits, after implementation staff left the setting, and in the face of competing pressures on clinic staff. We found that 
documentation was particularly difficult to maintain. I think it is fair to say, in hindsight, that we probably picked one of the worst possible times to take on this implementation challenge. We were in the midst of intense competitive pressures, and we had just closed one of our two hospitals, which was very hard on the morale of the organisation. We had undergone severe reductions in nursing and support staff. We went through two support staff strikes while we were doing this. There were also multiple competing initiatives going on. We implemented an electronic medical record, which is a great thing, but hugely taxing for everyone concerned. We implemented customer direct access to increase the patient satisfaction, but decreased clinician satisfaction in a significant way. This was a time of low morale and high turnover. Since then, things have settled down considerably.

Maintaining and institutionalising TRAC Once we had implemented the TRAC program in a random half of the facilities and the research phase was concluding, the health plan began planning for how to expand and institutionalise the program. First, they appointed a smaller TRAC oversight committee with stakeholders that had greater authority and operational influence within the system. The physician prevention leader, Richard Bills, began serving actively and effectively as the champion for TRAC. Operational responsibility for the program and for training control facilities and retraining the early implementation facilities was formally assigned to the health education department along with staffing resources. Staff can now document all of the 4As in electronic medical record and feedback is being provided regularly. Compliance with the documentation process is still a problem, but it is improving. What has really given a boost to the program is that the health plan leadership then took the critically important step of designating cessation advice as one of its five priority clinical targets. As a result, facility managers are now accountable for implementing TRAC, and salary bonuses for clinicians and staff are tied to the tobacco advice measure for 1999 .

\section{Conclusion}

Kaiser Permanente Northwest is making terrific efforts to implement and expand the TRAC model, and deserves a great deal of credit in my view. Many challenges remain, however. For example, how do you keep the focus on the patient and not on the performance target? How do we maintain the clinical skills of staff and train new staff on an ongoing basis, because unfortunately, these basic counselling skills are still not a routine part of clinical training or practice in most other settings? Another question we are wrestling with now is how to adapt TRAC to specialty departments. I am also concerned about how to maintain TRAC as an organisational priority as clinical performance targets multiply. A particularly nagging question is how to serve the hard core smokers who repeatedly try and fail. We do have a step care program in which patients can be referred to a multisession group treatment $\operatorname{program}^{16}$ in which participants can receive either nicotine replacement or buproprion as a covered benefit. Clinicians indicate on the electronic referral if there are medication contraindications, and additional screening and training about medication use occurs as patients enter the groups. Patients can also sign up for a multisession telephone support program that includes pharmacological treatment. These more intensive programs work quite well, but as we know, most smokers want to quit on their own and are unwilling to use these types of programs. ${ }^{1}$ Finally, there is the really important and unanswered question of how clinicians and HMOs can help adolescents avoid or give up tobacco? We have several large scale teen tobacco intervention projects going as we speak, but we do not have any answers yet.

In conclusion, if you are planning to implement an organised systems approach ${ }^{17}$ for delivering tobacco control in routine care, here are a few steps to consider:

- establish a tobacco task force with high level stakeholders

- adopt performance quality targets for all of the 4As, not just "ask and advise"

- create efficient staff roles that fit into the office flow

- make documentation and referrals very convenient for staff

- report regularly on the performance of teams and the whole organisation

- make performance matter through bonuses, performance reviews, or both

- celebrate progress!

Many are contributing to the ongoing effort, but I especially want to recognise the extraordinary contributions of Richard Bills, Susan Caulfield, Leslie Culligan, Dawn Hayami, John Ketarkus, Kathy Mount, Jennifer Boon, Kimberly Smith, Janice Krumenacker, Belle Slesh, Nick Socotch, Nancy Stevens, Thomas Stibolt, and Milissa Weisensee, and, of course, the managers, clinicians, and staff of Kaiser Permanente Northwest. Support was provided by NHLBI (R01 HL50007) and Kaiser Permanente.

1 Lichtenstein E, Hollis JH, Severson HH, et al. Tobacco cessation interventions in health care settings: rationale, model, outcomes. Addict Behav 1996;21:709-20.

2 Fiore MC, Bailey WC, Cohen SJ, et al. Smoking cessation. Clinical Practice Guideline No 18. Rockville, Maryland: US Department of Health and Human Services, Public Health Service, Agency for Health Care Policy and Research, April 1996. (AHCPR Publication No 960692.); <http://www.text.nlm.nih.gov/ftrs/tocview>.

3 Cromwell J, Bartosch WJ, Fiore MC, et al. Costeffectiveness of the clinical practice recommendations in the AHCPR guideline for smoking cessation. $7 A M A$ the AHCPR guideli

4 Meenan RT, Stevens VJ, Hornbrook MC, et al. Costeffectiveness of a hospital-based smoking-cessation inter-

5 Hollis J. Population impact of clinician efforts to reduce tobacco. In: Burns DM, ed. Population Based smoking cessation monograph. National Cancer Institute and the California Department of Health Services, Tobacco Control Section. In press

6 Gilpin E, Pierce J, Goodman J, et al. Trends in physicians' giving advice to stop smoking, United States, 1974-87. Tobacco Control 1992;1:31-6.

7 CDC. Physician and other health care professional counselling of smokers to quit-United States, 1991. FAMA 1993;270:2536-7.

8 Tomar SL, Husten CG, Manley MW. Do dentists and physicians advise tobacco users to quit? $\mathcal{F} A D A$ 1996;127:25965.

9 Thorndike AN, Rigotti NA, Randall SS, et al. National patterns in the treatment of smokers by physicians. $\mathcal{F} A M A$ 1998;279:604-8.

10 Vogt TM, Hollis JF, Lichtenstein E, et al. The medical care system and prevention: the need for a new paradigm. HMO Practice 1998;12:5-13. 
11 Hollis J, Lichtenstein E, Vogt T, et al. Nurse-assisted counselling for smokers in primary care. Ann Intern Med 1993; selling for

12 Lichtenstein E, Hollis J. Patient referral to a smoking cessation program: who follows through? $\mathcal{f}$ Fam Pract 1992;34:739-44.

13 Whitlock EP, Vogt TM, Hollis JF, et al. Does gender affect response to brief clinic-based smoking intervention? Am $\mathcal{F}$ Prev Med 1997;13:159-66.

14 Miller WR, Rollnick S. Motivational interviewing: preparing people to change addictive behavior. New York: The Guilford Press, 1991.

15 Rollnick S, Heather N, Bell A. Negotiating behavior change in medical settings: The development of brief motivational interviewing. 7 Ment Health 1992;1:25-37.

16 Stevens VJ, Hollis JF. Preventing smoking relapse using an individually tailored skills-training technique. $f$ Consult Clin Psychol 1989;57:420-4.

17 Solberg LI, Kottke TE, Conn SA, et al. Delivering clinical preventive services is a systems problem. Ann Behav Med preventive services

\section{Questions and answers}

$\mathrm{Q}$ : One of the issues that our organisation has been challenged with is trying to develop a system that addresses all prevention needs, because there are competing concerns. The challenge is to develop a system that is evidence based and addresses prevention globally.

A: We need to do a better job of integrating the roll out. I think your suggestion is on target; we need to be more organised in order to provide all the preventive services in an effective and synergistic way. Currently, staff have varying responsibilities, such as improving immunisations or improving screening. Some of the performance targets are more applicable to some areas of the health care system than others. Presently, programs are being rolled out independently, which is probably a mistake.

Q: We've heard about different types of incentives at this conference. We have heard about incentives that are performance based which allow for capitation bonuses based on meeting performance targets for tobacco. We have also heard about more organic incentives that stem from improving the quality of day-to-day work with one's colleagues, and with one's patients. Where are we in terms of understanding and using incentives to promote consistent tobacco intervention? Do you have any advice to help the field move forward?

A: There are, of course, many environmental reasons for doing what we do. Those which apply to smoking can be organised from the top down. There are the external incentives, or the extrinsic rewards such as pay. One level down from that is the incentive which I think is very, very powerful, that is the power of feedback and social comparison. We all like to feel like we are providing good medicine, or that we are performing state of the art service. Even without a financial tie to it, feedback itself is extraordinarily powerful, if it can be presented in a way that makes it clear how you are performing compared with others. People often feel they have the worst case mix, though, and there are extenuating circumstances at times, so there can be problems. Social comparison is very powerful, however. What will make this successful is systems that number one, work for the physician, and number two, work for the patient. There is an immediate intrinsic feedback that comes from a successful experience with patients. That kind of feedback will ultimately be the most powerful.

Q: You examined the structure of the managed care plan. How important is it to go beyond that for buy-in? At what level do we look for support? Should we go to purchasers and get them to support efforts for smoking cessation or to the public health officials at the state level?

A: The issue is, how do we change the culture of medicine so that this kind of activity is part and parcel of what we do? As purchasers start demanding cessation programs (it is one of the HEDIS measures), and as patients start to expect and value cessation activities, the climate will gradually change. There is generally resistance to cultural change, however. External incentives and monitoring functions help legitimise cessation programs, and this is an important part of what needs to occur. The primary power of the external incentives lies in the statement they make, that smoking cessation is important and we as a health care system recognise that. Thus, I do think purchasers have a lot of potential power in this arena which they have not yet exercised. 